\title{
Proficient Networking Protocol for BPLC Network Built on Adaptive Multicast, PNP-BPLC
}

\author{
Ali Md Liton, Zhi Ren, Dong Ren, Xin Su \\ School of Information and Communication Engineering Chongqing \\ University of Posts and Telecommunications, Chongqing, China
}

\begin{abstract}
In order to solve the problems of the existing broadband power line carrier communication standard IEEE1901.1 data link layer protocol network, multiple primary nodes receiving the beacon will send connotation entreaty message, and CCO will send an association sanction message proximately after receiving the message to confirm their character the central coordinator CCO association confirmation message reply is not timely to reduce the success rate of network access, high network access delay and control overhead. Based on the characteristics of BPLC carrier network, Proficient networking instrument of broadband PLC built on adaptive multicast PNP-BPLC is proposed in this paper. The simulation results show that adaptive multicast is used when CCO replies to the primary station, which can excellently recover the success rate of low-voltage PLB carrier communication nodes, decrease the network access delay and cut the network control overhead. Finally we used to OPNET simulation software to prove simulation result.
\end{abstract}

Keywords-Powerline communication; network delay; control overhead; central coordinator

\section{INTRODUCTION}

Low voltage broadband powerline carrier communication (BPLC) $[1,2,3]$ is a communication mode that uses lowvoltage power distribution line (380/220V subscriber line) as information transmission medium for voice or data transmission. It has the natural advantage that there is no need to reset up the network. The BPL carrier communication has wide bandwidth, and the basic frequency band is $1 \mathrm{MHz} \sim$ 20MHZ Compared with the traditional NBPLC carrier communication, Broadband PLC has higher transmission rate, stronger anti-interference performance and better performance. At present, the international standard for broadband power line carrier communication is IEEE 1901.1 [4-5]. This standard is created on Q/GDW 11612 technical description for interconnection and interworking of low voltage PLB carrier communication [5] of the state grid corporation of china, and grasps the operative tender of internet of things expertise based on power line carrier communication in energy internet [6]. BPLC has been widely used in automatic meter reading [7 8 9], intelligent power consumption system [8], street lamp control [8], charging pile construction [10 11], etc. At present, there are a lot of research in the field of BPLC technology. For example, literature $[10,11,12]$ explores the features of lowvoltage BPL carrier communication channel. For the input impedance features, signal noise and interloping characteristics, phase shift characteristics, the actual communication area of PLC is hundreds of meters. In order to confirm link stability and long distance transmission, literature [13-14] offers that tree networking and multi hop transmission similar to wireless sensor networks are assumed in the network management sublayer. Many people have planned the BPLC networking algorithm Literature [14-15] proposed an improved Q-learning algorithm suitable for multi restraints of PLC, LAN through incessant collaboration with the unknown situation. Although this kind of ant colony algorithm has certain adaptive ability, it needs to send a large number of control messages to interact with neighbor nodes, which has the problems of excessive control overhead and waste of resources. Reference [16-17] provides a tree based networking method for low voltage BPL carrier communication network, which uses topology assessment frame to travel the network topology, upholds and updates the sub node set according to the lowest energy value required for normal $[18,19,20]$ communication, picks candidate routing nodes in turn according to the energy value, and then assigns [24-25] network address to each sub node in the sub node set, Although this technique is simple and easy, it does not give the vigorous rebuilding and optimization manner of routing, and the network constancy is poor. IEEE1901.1 [21, 22, 23,] standard also espouses impart networking. The CCO elicits the network access appeal of STA level by level by sending the central beacon, placing the sending of discovery beacon and sending of proxy beacon to ample the entire networking procedure.

Rest of the paper we have described simulation results of the paper. In the Section of III(B)(1) we described, Network Access success rate, in Section III(B)(2) Average network access delay and in Section $\operatorname{III}(B)(3)$ Network control overhead, proposed PNP-BPLC Protocol is better than the other two protocols.

\section{System Model AND PROBlem Descriptions}

\section{A. Network Scenario}

The broadband carrier communication network will normally form a multi-layer tree network with the central coordinator CCO as the center and the proxy coordinator PCO as the relay agent to connect all stations STA. As shown in Fig. 1, the topology of a typical BPL carrier communication network is shown.

\section{B. Protocol Stack Structure of BPLC}

Based on the standard open system interconnection (OSI) seven layer model, the Broadband carrier communication network protocol stack defines three layers, physical layer, data link layer and application layer. 


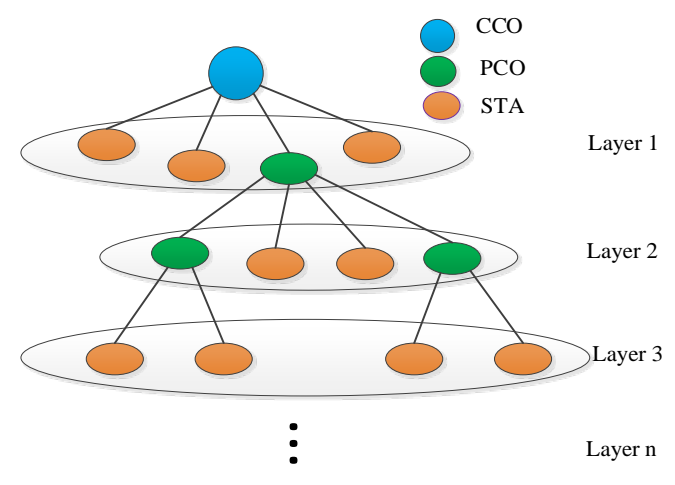

Fig. 1. Topology of BPL Carrier Communication Network.

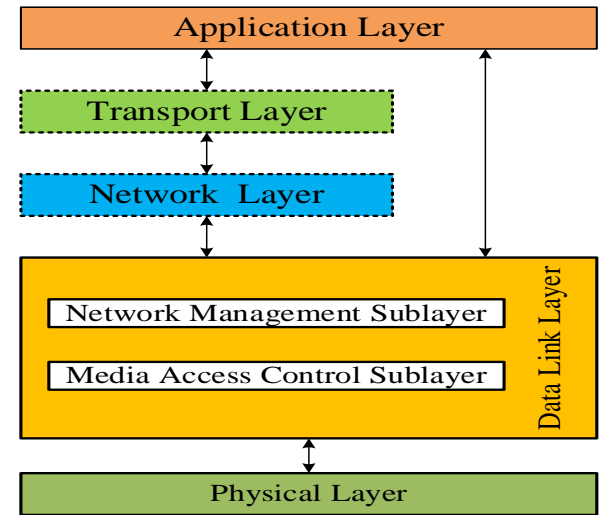

Fig. 2. BPLC Communication Network Protocol Stack.

The basic structure is shown in Fig. 2. The data link layer is divided into network management sublayer and media access control sublayer MAC sublayer. The data link layer directly provides transmission services for the application layer and can also be prolonged to edge with standard TCP / IP to grasp standard IP network communication.

\section{The BPLC Networking}

In order to certify the consistency of BPLC network routing and data spread, BPLC network will be networked. The procedure is as follows.

1) After $\mathrm{CCO}$ is powered on, coordinate the time slot and network documentation among networks. After effective organization, demeanor solitary network networking.

2) CCO starts to send the central beacon in the beacon slot, and the station receiving the central beacon wants to enter by sending the association appeal message in the CSMA slot access.

3) CCO verifies the site requesting network access through the white list. After positive confirmation, CCO sends the dispensation result to the STA site requesting network entree through the connotation sanction message, expressive the effective network access of the node.

4) After the primary node effectively arrives the network, CCO will arrange beacon time slot for it to send discovery beacon. The sending of discovery beacon can elicit the secondary site around the new site to recruit the request for related network access.
5) This rotation allows the main level STA site utmost from CCO to connection the network.

\section{Problem Description}

It is found that there are two problems in the BPLC networking progression.

1) In BPLC networking, after $\mathrm{CCO}$ sends the central beacon in the beacon slot, multiple primary nodes receiving the beacon will send connotation entreaty message, and CCO will send an association sanction message proximately after receiving the message to confirm their character. With the STA node layer by layer forwarding the association request message network entire tender through PCO, the number of STA nodes increases, and the association authorization message is likely to be hugged in the CCO queue, ensuing in the CCO inept to apportion TEI, time slot and other evidence to the nodes to be edited in time, and the network access solicitation node cannot access the network normally, resulting in the reduction of the network access attainment rate of BPLC network nodes.

2) STA sends a huge number of connotation entreaty messages, and CCO needs to send Association validation messages of the similar scale for retort. However, distinct beacon time slots, CSMA time slots assign superior time slots for separately node, so encounters must be dodged, ensuing in CCO's fiasco to reply relationship validation messages to overtone request messages sent by some nodes smearing for network access in time, then these STA,s will prompt the instrument of resending connotation request message because they cannot collect overtone sanction message within the waiting time edge Tmax, consequent in the rise of control overhead and network access delay.

\section{E. PNP-BPLC Mechanism}

In order to solve the problems described in the previous section, this paper offers a BPLC proficient Networking (PNP) mechanism built on adaptive multicast, including two new mechanisms adaptive multicast reply Association confirmation message and association reply based on retransmission message. The specific ideas are as follows.

\section{F. Adaptive Multicast Reply Connotation Confirmation Message Mechanism}

For the problem a) declared in the previous section, seeing that the BPLC is a tree topology, some STA sub sites will be equestrian under PCO, which means that the higher the level is, the more stations are likely to be. Then it is likely that the association confirmation messages sent by multiple websites to be accessed will be put into the cache queue by $\mathrm{CCO}$ and not replied in time. Therefore, this paper propositions CCO adaptive multicast replies Association sanction message apparatus.

The core idea is CCO queries the number of association confirmation messages in its queue. When the number is1, unicast Association confirmation messages. When the number is greater than1, multicast association instant warning messages are sent to send association reply messages more 
rationally, which increases the network access success rate of nodes and condenses the network access delay and control overhead. Since multilevel nodes will also onward the association request message to the prime node, which is principally the same as that of the prime node, this paper mainly discusses the association retort of CCO to the principal node. The basic process is as follows.

Step 1: Start the neighbor network observing timer when $\mathrm{CCO}$ is drove on. If the inter network direction frame is received within the listening time $t$, coordinate the inter network identification NID and time slot. If the inter network direction frame is not received within the listening time t, single network networking is carried out. CCO recordings the central beacon in the beacon slot.

Step 2: The neighbor node receives the central beacon, forms whether the "start association flag bit" of the beacon is 1 , and is ready to send the association entreaty message, before the station is ready to send, listen for data being transmitted on the bus whether the line is busy or not. If the line is found to be busy during listening, wait for a delay and listen again. If it is still busy, continue to delay the wait. If the waiting times $n$ exceed the threshold 16, STA retransmits the association entreaty message and marks the message. If the time of each delay is erratic, it is resolute by the reduced two the M-ray exponential bakeoff algorithm fixes the delay value.

Step 3: CCO receives the association request message sent by the node relating for network access, inquiries the STA site information consistent to the association request message, and completes white list confirmation. CCO queries the number of layers of the site and regulates the terminal address of the next hop. If it is a first class site, the destination address is the node. If it is a multi-layer site, the destination address is the PCO of the lowest level agreeing to the inviting site. CCO allocates TEI and time slot to the node and keeps them in the reminder reply message. CCO makes association reply to the principal site. If the channel is busy when sending Association reply, CCO will put the association reply message into a distinct association reply backlog.

Step 4: Check the number of association retorts in the queue. If it is 1 , wait for the channel idle unicast to send the association sanction message. If it is greater than 1 , all overtone messages in the queue are taken out, the address evidence of each node is found, and the association rapid hint message is formed. When the channel is idle, multicast is sent to the crucial node.

Step 5: The primary node limits whether it is the node applying for network access. If so, the network entree is effective. If not, the node is the lowest level PCO of the node applying for network access. The node forms an association endorsement message through the association reply message sent by CCO to itself and sends it to the next level node. The next level node also benches whether it is the node applying for network access first. If not, the node is the lowest level PCO of the node applying for network entree.

Then the node is the secondary PCO of the network access node. The node installs the address evidence and remains to send the association sanction message to its subsidiary nodes, and so on until the association request node is found and the network entrée is effective.

\section{G. Association Reply Apparatus Based on Retransmission Message}

In order to solve the problem b), this paper propositions an association reply apparatus based on retransmission message. Its core idea is perceptive.

CCO adaptively sends the first level reply message by arbitrating whether the retransmitted association request message has been received around. If CCO receives the association request message retransmitted from STA node, it specifies that the association request message formerly sent by STA node has not received Association confirmation, which tortuously indicates that the BPLC network channel is busy.

If CCO remains to unicast Association credit messages in CSMA time slots, the channel cramming will be impaired. CCO stops generating association confirmation message unicast transmission and selects to verify the received association request message, and generates association rapid hint message, so that there is no large number of association confirmation messages competing with association request messages in CSMA time slot, so as to slow down the message load of the channel and reduce the control overhead.

Fig. 4 is 1 graphic diagram of association reply apparatus built on retransmission message. Fig. 4(1) shows the uplink association request message sent by STA and the association sanction message sent by CCO under normal conditions. Fig. 4(2) shows the graphic diagram of more network access nodes. As can be seen from the figure, due to the large number of nodes, both uplink and downlink messages need to compete for channels, resulting in the STA sending the uplink Association reply message represented by the blue arrow not receiving the agreeing association confirmation message and not being able to access the network normally. After a waiting time, the association request message with the green arrow is sent again. Fig. 3 is the graphic diagram of the new apparatus, and the red arrow represents the association instant indication message sent by CCO multicast. In Fig. 3, after CCO senses that some STA resend association request messages, it uses the method of ending sending Overtone sanction messages and multicast Overtone instant indication messages, which almost reduces the control overhead by $50 \%$ and importantly cuts the drain of the channel.

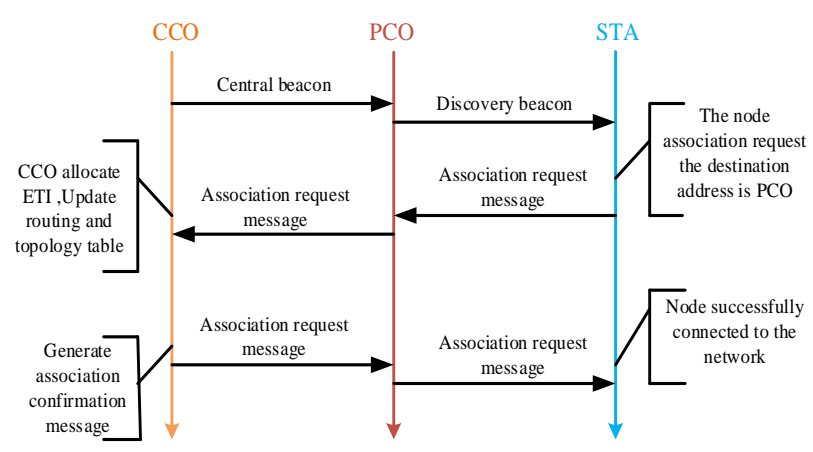

Fig. 3. Message Interaction of Associated Network for BPLC. 


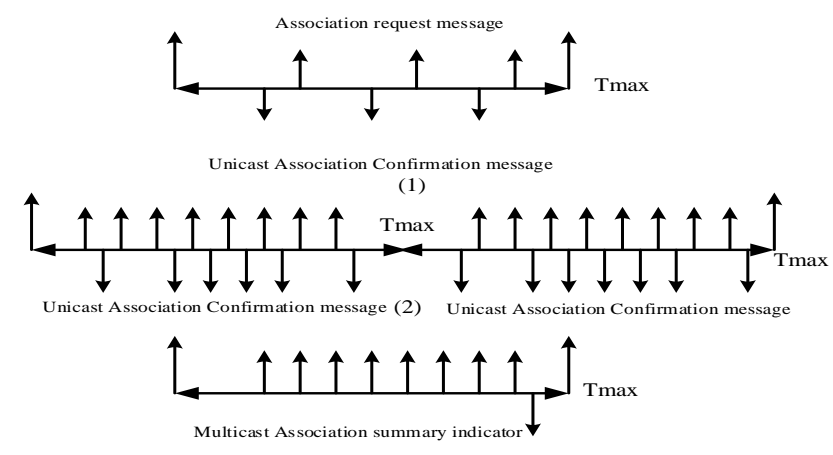

(3)

Fig. 4. Association Reply Apparatus based on Retransmission Message.

The Basic Operation Process is as follows:

Step 1: CCO performs network organization, which is reliable with CCO in the adaptive multicast reply association confirmation message device.

Step 2: STA makes association request, which is consistent with the association request made by STA in the adaptive multicast reply Connotation confirmation message apparatus.

Step 3: CCO receives the association request message sent by STA and benches whether there is a retransmission flag. If so, it stops sending the connotation confirmation message. In this time slot, it only accepts the association request message, takes out the node ID, original address and other information, and forms an association instant hint message. Multicast sends Association instant indication message at Tmax time. The algorithm of multicast sending association rapid hint message is consistent with that of adaptive multicast reply connotation confirmation message apparatus.

Step 4: The main node regulates whether it is the node smearing for network access. If so, the network access is effective. If not, the node is the lowest level PCO of the node smearing for network access, forming an association confirmation message. This procedure is constant with the progression in which the node judges whether it is the node smearing for network access in the adaptive multicast reply association confirmation message apparatus.

\section{H. PNP-BPLC Apparatus Progression}

The flow chart of BPLC proficient networking apparatus based on adaptive multicast is shown in Fig. 5, which includes two new mechanisms adaptive multicast reply association confirmation message and association reply built on retransmission message.

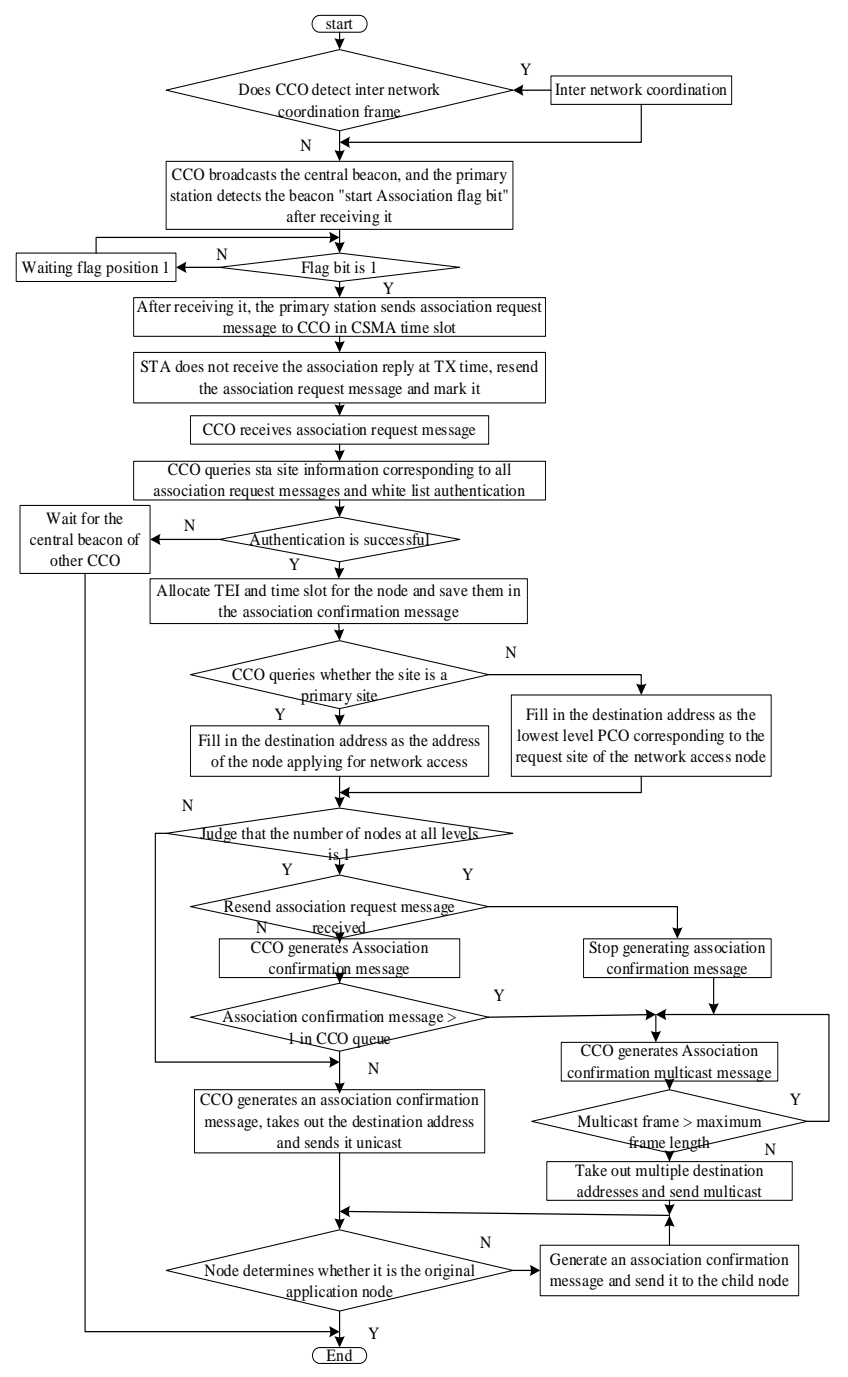

Fig. 5. Adaptive Multicast Access Protocol Process.

\section{SIMULATION ANALYSIS}

\section{A. Simulation Parameter Setting}

This paper uses OPNET 14.5 simulation tools to simulate IEEE1901.1 technical standard and Literature [15], PNP-BPLC apparatus are used to simulate the network access success rate, average network access delay and control overhead. The main simulation parameters are shown in Table I.

TABLE I. MAIN PARAMETER SETTING

\begin{tabular}{|l|l|}
\hline Simulation Parameter & Value \\
\hline Simulation scene $/ \mathrm{m} * \mathrm{~m}$ & $3000 * 1000$ \\
\hline Simulation running times/s & 100 \\
\hline Number of nodes/Pc & {$[4,9,14,19,24]$} \\
\hline Transmission rate /Mbps & 10 \\
\hline Single level network access time/s & 20 \\
\hline Single hope effective transmission distance /M & 500 \\
\hline $\begin{array}{l}\text { Number of CCO } \\
\text { Maximum hops/hop }\end{array}$ & 1 \\
\hline
\end{tabular}




\section{B. Performance Index}

1) Network access success rate: Indicates the network access success rate of each STA site of BPL carrier communication network. The network access success rate $\mathrm{P}$ is calculated as Formula 1.

$p=\sum_{i=1}^{i=n} X i \div \sum_{i=1}^{i=n} Y i \times 100 \%$

Where $\mathrm{n}$ represents the simulation scenario with $\mathrm{n}$ nodes, $\mathrm{Xi}$ represents the number of association request messages sent by the ith node, and Yi represents the number of association confirmation messages received by the ith node.

2) Average network access delay: The average network access delay represents the generation time TpK from the creation of the central beacon frame the average time of Tsim when the association reply message sent by the create to the CCO is received by the node applying for network access. With transmission delay, spread delay, inter layer processing delay and MAC layer queuing delay. The average network access delay is calculated according to formula 2 .

$\bar{T}=\frac{1}{n} \sum_{i=1}^{i=n}\left(\mathrm{~T} \operatorname{sim}(\mathrm{i})-\mathrm{Tpk} \_\right.$creat(i) $)$

3) Network control overhead: Network Control overhead refers to the sum of bits of control message required in the whole networking process of BPLC network. In BPLC network, there are four types of control messages inter network coordination message, beacon message, association request message and association reply message. Because the simulation is in a single network environment, the overhead message in this paper does not include inter network coordination message. Among them, the beacon message is divided into central beacon, proxy beacon and discovery beacon. The association reply message also includes association validation message and association summary indication message. The calculation of BPLC control cost C is shown in Formula 3.

$C=8 *\left(M *\left(c_{11}+c_{12}+c_{13}\right)+N^{*} c_{2}+O^{*}\left(c_{31}\right)+P\left(c_{32}\right)\right)$

Among them, C11, C12, C13, C2, C31 and C32 are the number of central beacon, proxy beacon, discovery beacon, overtone request message, association confirmation message and association rapid indication message singly, which can be counted by simulation. M. N, O and P, are beacon frames, association request message, association validation message and association instant indication message, respectively. According to IEEE 1901.1 standard, MAC frame is the basic transmission unit for transmission between MAC layers of different stations. A MAC frame consists of MAC frame header, MAC service data unit MSDU and integrity check value. In this paper, the size of long frame header is $h=26$ bytes and the size of integrity check value is $\mathrm{w}=4$ bytes.
The beacon frame size $\mathrm{M}$ is calculated according to formula 4 .

$M=h+m+w$

$\mathrm{M}$ is beacon frame MSDU size $=$ MPDU frame control size 16 bytes + frame load 520 bytes $=536$ bytes. Thus, $M=576$ bytes can be obtained.

The calculation of association request message $n$ is shown in formula 5.

$N=h+n+w$

$\mathrm{N}$ is the MSDU size of association request message $=4$ bytes of Association message header +64 bytes of association request message format $=68$ bytes. It can be concluded that $n=$ 98 bytes.

The calculation of association confirmation message $\mathrm{O}$ is shown in formula 6 .

$O=h+O+w$

$\mathrm{O}$ is the MSDU size of association confirmation message = 4 bytes of association message header +64 bytes of association confirmation message format $=68$ bytes. It can be concluded that $0=98$ bytes.

The calculation of association request message $\mathrm{P}$ is shown in formula 7.

$P=h+p+w$

$\mathrm{P}$ is the size of MSDU of association instant hint message = 4 bytes of header of association message + format of association summary indication message q, q = basic length of association instant indication message + extended variable length.

It is assumed that the maximum threshold of association confirmation messages queued in CCO queue at the same time is 10 . Therefore, $\mathrm{q}$ is the maximum number of fills $10 *$ each site information field, as shown in Table II, the size is 8 bytes = 80 bytes. Therefore, $\mathrm{P}=84+30=114$ bytes.

TABLE II. SITE INFORMATION FIELD

\begin{tabular}{|c|c|c|c|c|}
\hline Field & Byte number & Bits & Field size & Definition \\
\hline $\begin{array}{l}\text { Site address } \\
1\end{array}$ & $0-5$ & $0-7$ & 6 bytes & $\begin{array}{l}\text { MAC } \\
\text { address }\end{array}$ \\
\hline Site ETI 1 & 6 & $0-7$ & 12 bits & $\begin{array}{l}\text { TEI assigned } \\
\text { to site }\end{array}$ \\
\hline Site ETI 1 & 7 & $0-3$ & Site TEI 1 & $\begin{array}{l}\text { TEI assigned } \\
\text { to site }\end{array}$ \\
\hline Retain & 7 & $4-7$ & 4bits & Retain \\
\hline ......... & ......... & .......... & ......... & .......... \\
\hline $\begin{array}{l}\text { Site } \mathrm{n} \\
\text { address }\end{array}$ & $\cdots \cdot$ & & $\ldots$ & $\begin{array}{l}\text { Site } \mathrm{n} \\
\text { address }\end{array}$ \\
\hline Site TEI n & ........... & & $\ldots$ & $\begin{array}{l}\text { TEI n=Total } \\
\text { number of } \\
\text { stations }\end{array}$ \\
\hline
\end{tabular}


It can be proved theoretically that the message overhead using PNP apparatus is less than that of the original BPLC network access apparatus.

Condition (1): Except for the associated reply message, the overhead of other control messages is equal and is not counted

Condition (2): Number of messages in CCO Association confirmation queue $\mathrm{m}>=1$.

Prove: The message overhead of PNP apparatus is less than that of the original BPLC network access apparatus.

Multicast association summary indication message overhead $\mathrm{C} 2=8 *(\mathrm{H}+8 * \mathrm{~m}+\mathrm{W})=(\mathrm{b} 4 \mathrm{~m}+240)$ bit using the PNP mechanism; because $\mathrm{m}>=1$; Therefore, C1-C2 = $784 \mathrm{~m}-(64 \mathrm{M}+240)=720 \mathrm{~m}-240>=480$ bit $>0$; Get a certificate.

Using the original PLC networking apparatus, unicast association confirmation message overhead $\mathrm{C} 1=8 *(\mathrm{~m} *(\mathrm{H}+$ $\mathrm{O}+\mathrm{W}))=784 \mathrm{mbit}$;

\section{Analysis of Simulation Results}

1) Network access success rate: As shown in Fig. 6, the PNP-BPLC contrivance and IEEE1901.1, Literature [15] simulation comparison diagram of data link layer protocol data network access success rate. It can be seen that with the increase of the number of stations and network level, the network access success rate of the two mechanisms gradually decreases, which is due to the increase of channel load and the repeated retransmission of association request message through the comparison of the two figures.

It can be seen that when there are few nodes, the network access success rates of the two mechanisms are basically the same. With the increase of the number of nodes, the network access success rate of PNP device is higher than that of IEEE190.11 and Literature [15] network access appliance of 1 MAC protocol. Because the BPLC network is a multi-level and multisite network, the two improved methods proposed by PNP-BPLC contrivance successfully improve the network access success rate of BPLC network.

2) Average network access delay: As shown in Fig. 7, the PNP-BPLC device and IEEE1901.1, Literature [15] comparison diagram of network access delay of different node scenarios in 100s simulation time of 1 MAC layer protocol network access mechanism. Through comparison, it can be seen that with the increase of the number of nodes, the amount of data in the network gradually increases, resulting in the gradual increase of the average network access delay of nodes. The network access delay of PNP-BPLC appliance is lower than IEEE1901.1, Literature [15] the network access delay of 1 MAC layer protocol network access mechanism, and the advantages become more obvious with the number of nodes. Description in IEEE1901.1, Literature [15] under the standard, with the increase of the number of nodes, many association confirmation messages are not sent in time in the CCO queue. It increases the network access delay. The PNP-BPLC contrivance reduces the transmission of control messages, reduces the congestion of the channel almost.

3) Network control overhead: As shown in Fig. 8, control overhead comparison diagram shows the PNP-BPLC and the original PLC network access mechanism IEEE1901.1, Literature [15] the comparison diagram of the control overhead of the standard network access mechanism can clearly see that the control overhead of the two apparatuses increases steadily when the BPLC enters the network step by step, because with the increase of nodes, more nodes need to send control messages, which upsurges the control overhead. Through comparison, it is obvious that the control overhead of PNP-BPLC appliance is basically the same as that of the original PLC network access mechanism when the number of nodes is small. This shows that when the number of nodes is small and the channel is idle, the reply of CCO to the network access node is mainly association confirmation message. However, in the multi-layer PLC network environment with a large number of nodes, the control overhead of the original PLC network access mechanism increases almost exponentially, while the control overhead of PNP-BPLC mechanism increases only linearly.

Depiction in IEEE 901.1, Literature [15] standard, with the growth of the number of nodes, many association confirmation messages are not sent in time in the CCO queue, resulting in retransmitted association request messages, which greatly increases the control overhead of the network. The PNP-BPLC mechanism can adaptively send the association reply message, which greatly reduces the overhead of the association confirmation message sent by the original CCO. At the same time, it also reduces the retransmission of the association request message by the network access application node, and reduces a large number of message overhead.

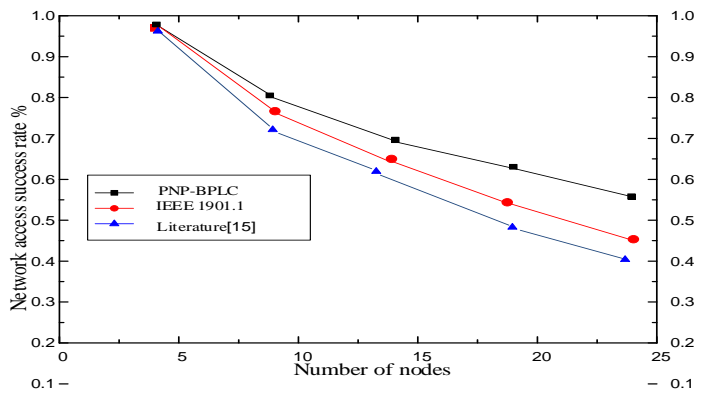

Fig. 6. Comparison of Success Rate of Network Access.

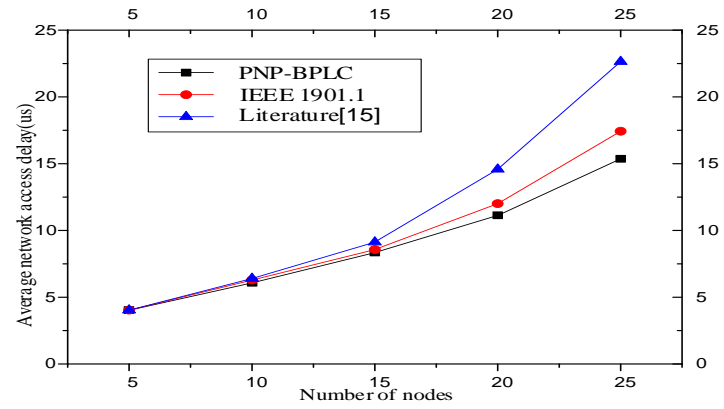

Fig. 7. Comparison of Average Network Access Delay. 


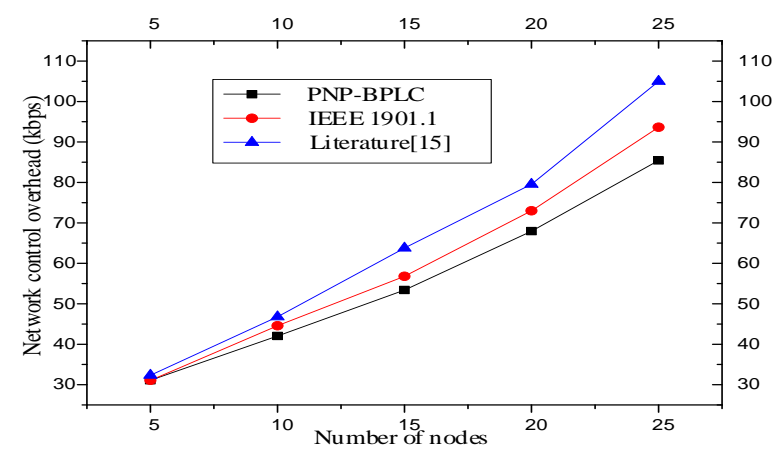

Fig. 8. Comparison of Network Control Overhead.

\section{CONCLUSION}

The low voltage PLC communication network is affected by robust interference, which requires the networking process to have high adaptive ability. Aiming at the two problems of BPLC data link layer networking, combined with the BPLC network scenario, this paper propositions proficient BPLC networking apparatus built on adaptive multicast. It includes two new mechanisms adaptive multicast reply association confirmation message and association reply based on retransmission message. By sending the primary association reply message more reasonably, the channel resources are more reasonably utilized, and the BPLC networking efficiency is higher. The experimental results show that the above mechanism can improve the data access success rate of BPLC network, reduce the access delay, and greatly reduce the control overhead of multi-layer BPLC network, which verifies the effectiveness of PNP-BPLC mechanism.

\section{ACKNOWLEDGMENT}

This work was supported in part by the National Natural Science Foundation of China (61971080), the Key Project of Fundamental and Frontier Research of Chongqing (cstc2015jcyjBX0085), and the cooperative project of Chongqing Electricity Research Institute of State Grid of China (SGCQDK00NYJS1900346.

\section{REFERENCES}

[1] Zhang Hao. Research on data link layer protocol simulation and performance of broadband power line communication system [D]. Xi'an University of Electronic Science and technology, 2017.

[2] H. Hrasnica, A. Haidine, R. Lehnert, “ Broadband Powerline Communications Network Design, ” Willey , c2004. 275 s. ISBN 0-47085741-2.

[3] Galli S, Scaglione A, Zhifang W. For the grid and through the grid: the role of power line communications in the smart grid. P IEEE 2011; 99: 998 -1027.

[4] Massaki K. Introduction to robust, reliable and high-speed power-line communication systems. IEICE T Fund Electr 2001; 12: 2958-2965.

[5] High Definition Power Line Communication (HD-PLC), [Online]. Available: http://www.hd-plc.org/

[6] UPA Digital Home Specification (DHS), [Online]. Available: www.upaplc. org
[7] Guzelgoz, S. (2011). Characterizing wireless and power line communication channels with applications to smart grid networks. $\mathrm{PhD}$ Dissertation, University of South Florida.

[8] IEEE Standard 1901 Power Line Networks: Medium Access Control and Physical Layer Specifications.

[9] L. Zhang, X. Liu, and D. Xu, “A novel security monitoring system of coal mine based on power line communication dynamic routing technology," in Industry Applications Society Annual Meeting, 2014

[10] S. Galli and T. Lys, "Next generation narrowband (under $500 \mathrm{kHz}$ ) power line communications (PLC) standards," China Commun., vol. 12, no. 3, pp. 1-8, 2015.

[11] Chen Feng, Zheng-Wengang, Shen Chang-jun, et al. Low voltage power line carrier communication technology and application [J]. Power system protection and control, 2009,37 (22): 188-195.

[12] IEEE Standard for Medium Frequency (less than $12 \mathrm{MHz}$ ) Power Line Communications for Smart Grid Applications," in IEEE Std 1901.12018 , vol., no., pp.1-192,14May2018doi:10.1109/IEEESTD. 2018.8360785.

[13] Q / GDW 11612-2016, technical specification for interconnection and interworking of low voltage power line broadband carrier communication [S].

[14] Fan yun-nian. Energy Internet, Qugao is not harmonious [n]. China Science Daily, April 23, 2020 (006).

[15] Duke, Wang Wei-jie, Mei Ping. Application of OFDM based lowvoltage power line broadband carrier communication technology in lowvoltage centralized reading system [J]. Technology outlook, 2016,26 (35): 83-84

[16] Lv Wei-jia. Application analysis of broadband power carrier technology in intelligent power system [a]. Proceedings of 2017 smart grid development seminar [C]. China Electric Power Research Institute: Taiji Computer Training Center, Haidian District, Beijing, 2017:5.

[17] Tong Shi-wei. Research on channel characteristics test and modeling of broadband power line based on street light line [D]. North China Electric Power University, 2019.

[18] Tan Bao-qi. Application of PLC technology of power carrier communication in the construction of charging pile [J]. China Equipment Engineering, 2019 (22): 161-162.

[19] [28] Yan Yuan-zhi. Analysis of channel characteristics of low voltage power line carrier communication [J]. Communication world, 2018 (08): 160-161

[20] Pan Dong-yang. Research on OFDM based low-voltage power line carrier communication system [D]. Chongqing University of technology, 2019.

[21] Zhang Jie. Research on Routing Technology Based on broadband power line carrier communication [a]. Proceedings of 2018 smart grid information construction seminar [C] of China Electric Power Research Institute. China Electric Power Research Institute: the Sixth Research Institute of China Electronic Information Industry Group Co., Ltd., 2018:4.

[22] Guo pan.Methods,devices,equipment, concentrators and systems for obtaining routes [P]. Cn102970233a, March 13, 2013.

[23] Li Gui-lin, Wei Sheng-qing. A tree based networking method for lowvoltage power line broadband carrier communication [P]. Cn107332777a, 2017-11-07.

[24] Long Yu-li. Form of relay network for power line carrier communication in low voltage distribution network [J]. Electronic technology and software engineering, 2018 (20): 41.

[25] Guangyu Pei and C. Chien. Low Power TDMA in Large Wireless Sensor Networks. In Military Communications Conference, 2001. MILCOM 2001. Communications for Network-Centric Operations: Creating the Information 